\title{
Cyber-Physical Systems Virtual Organization: Active Resources
}

\author{
Enabling Reproducibility, Improving Accessibility, and Lowering the Barrier to Entry \\ Stephen A. Rees \\ Vanderbilt University \\ Nashville, Tennessee \\ stephen.a.rees@vanderbilt.edu \\ Taylor T. Johnson \\ Vanderbilt University \\ Nashville, Tennessee \\ taylor.johnson@vanderbilt.edu \\ Tamas Kecskes \\ Vanderbilt University \\ Nashville, Tennessee \\ tamas.kecskes@vanderbilt.edu \\ Katie Dey \\ Vanderbilt University \\ Nashville, Tennessee \\ katie.dey@vanderbilt.edu \\ Patrik Meijer \\ Vanderbilt University \\ Nashville, Tennessee \\ patrik.meijer@vanderbilt.edu \\ Paulo Tabuada \\ University of California \\ Los Angeles, California \\ tabuada@ucla.edu \\ Marcus Lucas \\ University of California \\ Los Angeles, California \\ maluc@g.ucla.edu
}

\begin{abstract}
The Cyber-Physical Systems Virtual Organization (CPS-VO) ${ }^{1}$ has been evolving from a shared repository of information into a destination for active collaboration, simulation, hands-on education, and demonstration. We would like to show-case advances in tool integration, particularly a set of verification tools, and how this integration enables reproducibility, improves accessibility, and lowers the barrier to entry in this field. We would also like to demonstrate use of our simulation and tool frameworks, have a poster showing results and progress over the last year, and invite others to host their tools on the CPS-VO infrastructure.
\end{abstract}

\section{CCS CONCEPTS}

- Computing methodologies $\rightarrow$ Modeling and simulation; • Software and its engineering $\rightarrow$ Software libraries and repositories; • Mathematics of computing $\rightarrow$ Mathematical software; - Information systems $\rightarrow$ Web applications; • Applied computing $\rightarrow$ Education.

\section{KEYWORDS}

verification, simulation, modeling, reproducibility, analysis, webbased tools, educational tools, collaborative design, cloud services

\section{ACM Reference Format:}

Stephen A. Rees, Tamas Kecskes, Patrik Meijer, Taylor T. Johnson, Katie Dey, Paulo Tabuada, and Marcus Lucas. 2019. Cyber-Physical Systems Virtual Organization: Active Resources: Enabling Reproducibility, Improving Accessibility, and Lowering the Barrier to Entry. In 10th ACM/IEEE International

\footnotetext{
$\overline{{ }^{1} \mathrm{https} / / \mathrm{cps}-\mathrm{vo} . \mathrm{org}}$
}

Permission to make digital or hard copies of part or all of this work for personal or classroom use is granted without fee provided that copies are not made or distributed for profit or commercial advantage and that copies bear this notice and the full citation on the first page. Copyrights for third-party components of this work must be honored

For all other uses, contact the owner/author(s).

ICCPS '19, April 16-18, 2019, Montreal, QC, Canada

(c) 2019 Copyright held by the owner/author(s)

ACM ISBN 978-1-4503-6285-6/19/04.

https://doi.org/10.1145/3302509.3313331
Conference on Cyber-Physical Systems (with CPS-IoT Week 2019) (ICCPS '19), April 16-18, 2019, Montreal, QC, Canada. ACM, New York, NY, USA, 2 pages. https://doi.org/10.1145/3302509.3313331

\section{INTRODUCTION}

The Cyber-Physical Systems Virtual Organization (CPS-VO) was designed and built to serve as a repository of relevant information, intended to bring industry, academia, and government together across the field of cyber-physical systems, so that researchers could benefit from the sharing and dissemination of their data amongst each other. As this goal has been realized over the last few years, the scope of what the CPS-VO could do has expanded from being a static resource, to what we term an active resource. No longer just a portal where information can be gleaned, the CPS-VO is becoming a destination for engaging in activities.

Three main thrusts of the "active resources" effort were previously introduced: tool libraries, integrated tools, and design studios. The heaviest areas of focus in the last year have been the tool libraries, and the integration of some of those tools into design studios. These design studios offer a collaborative, versioned environment that is flexible, powerful, and light-weight.

\section{DESIGN STUDIO ARCHITECTURE}

The architecture for including design studios in the CPS-VO was developed with the intent of being simple to integrate and deploy, while placing low load on the CPS-VO server and appearing seamless to use. The need to separate the services of the CPS-VO from the run-time of individual tools for security and stability is obvious, so it was determined to establish instances of tools with a cloud provider, and integrate them into the CPS-VO via IFrames. The main hurdle then became unifying the authentication mechanism. We built a module for the CPS-VO to address authenticated transfers between webservers via two different methods: JSON web tokens $(\mathrm{JWT})^{2}$, and a small, flexible, shared-secret webhook. Since JWT are

\footnotetext{
${ }^{2}$ https://jwt.io/
} 
an open, industry standard method for representing claims securely between two parties, this is the preferred method.

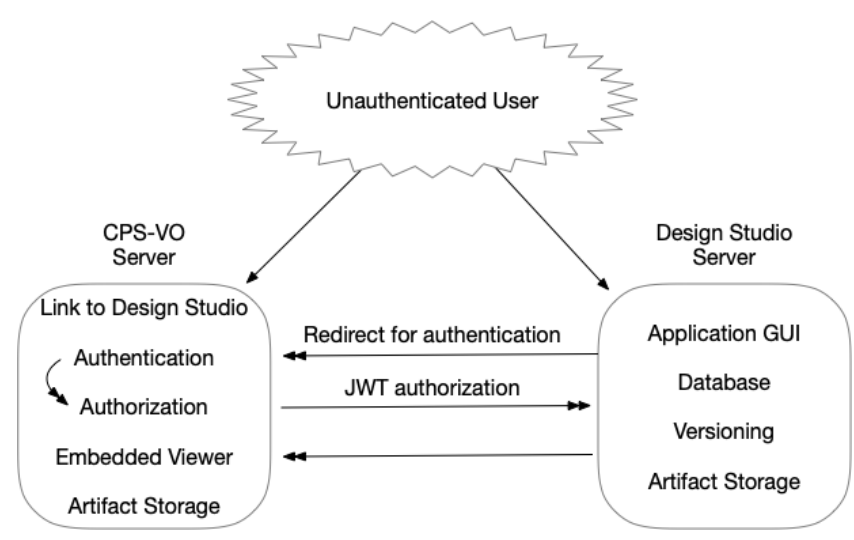

Figure 1: Integration via JWT

Figure 1 above shows the flow followed by an unauthenticated user who wishes to utilize a design studio integrated via JWT on the CPS-VO. Unauthenticated users who follow a link directly to the cloud-provisioned design studio server will be redirected back to an appropriate page on the CPS-VO about the design studio. This page will contain a link to a webhook on the portal which will first give the user a chance to authenticate with the CPS-VO, then determine if they are authorized to utilize the design studio, and if so, forward them to the design studio using a JWT to inform the design studio server of the user's credentials. The design studio provides a fully self-contained application that utilizes the transferred credentials to provide a private workspace for the user, which is then displayed seamlessly inside the CPS-VO portal through a shadowbox IFrame. Results of the tools run inside the design studio can be configured to be returned directly to the user from the design studio, or if integrated more deeply with the CPS-VO, can be stored automatically inside the CPS-VO repositories.

\section{DEMONSTRATION}

For our demo we would like to showcase a new design studio that integrates multiple verification tools into the CPS-VO. The goal of this studio is to contain a sufficient subset of verification tools to have a broad impact on the verification community. Making these tools available in a single design studio will improve the accessibility of each tool by removing the need for an end-user to install the appropriate environment. These tools will be easy to access and use, and give reproducible results - facilitating tool visibility, education, research, and community events such as the ARCH workshop's friendly competition ${ }^{3}$. A further benefit of this studio is that new verification tools can be seamlessly integrated without additional work on the studio, if they accept one of the supported exchange formats.

The core of this design studio is based on $\mathrm{HyST}^{4}$ running inside WebGME $^{5}$. The architecture of this design studio is demonstrated in

\footnotetext{
$\overline{{ }^{3} \text { https://cps-vo.org/group/ARCH }}$

${ }^{4} \mathrm{http}: / /$ verivital.com/hyst

${ }^{5}$ https://webgme.org/
}

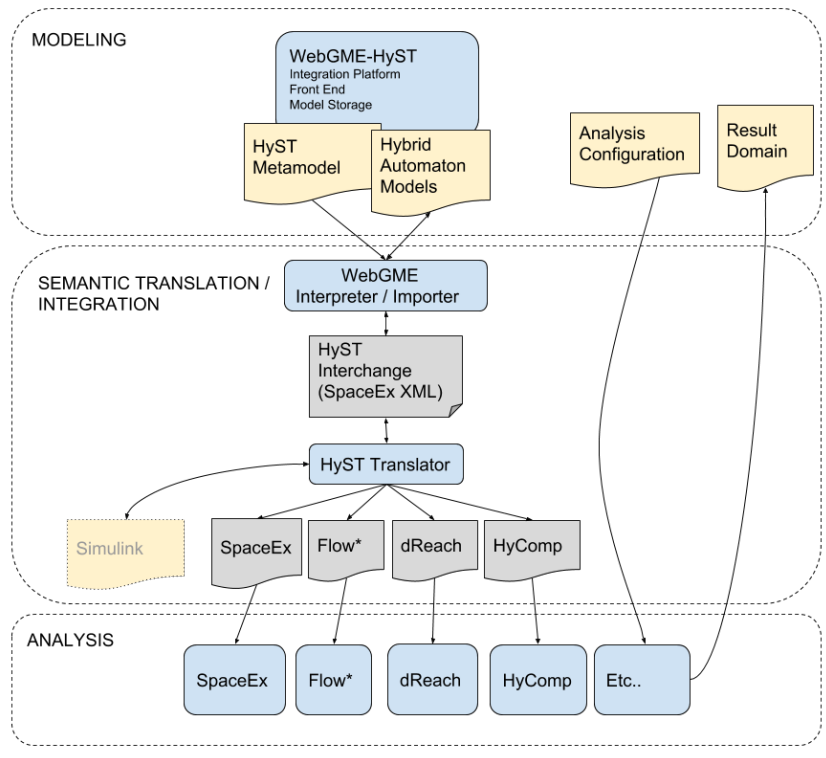

Figure 2: CPS-VO Verification Tool Design Studio

Figure 2. HyST provides a path for translating the SpaceEx ${ }^{6}$ model format into a handful of other formats necessary for analysis by their respective tools. Furthermore, this design studio can generate a SpaceEx model from its own native model as well as import directly from a pre-existing SpaceEx model. Since the CPS-VO already has a SpaceEx integration, users can choose if they would like to model in SpaceEx and import, or simply model inside WebGME. When modeling is complete, the user will be able to run a verification analysis from a selection of tools. The WebGME instance will spawn a Docker ${ }^{7}$ container of that particular verification tool and return the results to the user when analysis is complete. The Docker instance returns to its original state, enabling consistent reproducibility.

Attendees will be able to experiment with model import, building, and analysis execution, discuss and view how to integrate their own tools, view the current status of last year's OpenUAV[1] demo, and explore our library of tools. A pair of screens would be beneficial, as would a strong wireless or wired Internet connection.

\section{ACKNOWLEDGMENTS}

We gratefully acknowledge NSF grant CNS1521617 for supporting this work.

\section{REFERENCES}

[1] Anna Lukina, Arjun Kumar, Matt Schmittle, Abhijeet Singh, Jnaneshwar Das, Stephen Rees, Christopher P. Buskirk, Janos Sztipanovits, Radu Grosu, and Vijay Kumar. 2018. Formation Control and Persistent Monitoring in the openUAV Swarm Simulator on the NSF CPS-VO. In Proceedings of the 9th ACM/IEEE International Conference on Cyber-Physical Systems (ICCPS '18). IEEE Press, Piscataway, NJ, USA, 353-354. https://doi.org/10.1109/ICCPS.2018.00050

\footnotetext{
${ }^{6} \mathrm{http}: / /$ spaceex.imag.fr

${ }^{7}$ https://www.docker.com/
} 